PROCEEDINGS OF THE

AMERICAN MATHEMATICAL SOCIETY

Volume 138, Number 9, September 2010, Pages 3277-3284

S 0002-9939(10)10382-7

Article electronically published on April 16, 2010

\title{
GENERIC PROPERTIES OF CRITICAL POINTS OF THE SCALAR CURVATURE FOR A RIEMANNIAN MANIFOLD
}

\author{
ANNA MARIA MICHELETTI AND ANGELA PISTOIA
}

(Communicated by Matthew J. Gursky)

\begin{abstract}
Given $(M, g)$ a smooth compact Riemannian $N$-manifold, we prove that for generic Riemannian metric $g$ the critical points of the scalar curvature are nondegenerate.
\end{abstract}

\section{INTRODUCTION}

Let $M$ be a connected compact $C^{\infty}$ manifold of dimension $N \geq 2$ without boundary. Let $\mathcal{M}^{k}$ be the set of all $C^{k}$ Riemannian metrics on $M$. Any $g \in \mathcal{M}^{k}$ determines the scalar curvature $S_{g}$ of $(M, g)$. Our goal is to prove that for generic Riemannian metric $g$ the critical points of the scalar curvature $S_{g}$ are nondegenerate. More precisely, we will prove the following result.

Theorem 1.1. The set

$$
\mathcal{A}:=\left\{g \in \mathcal{M}^{k}: \text { all the critical points of } S_{g} \text { are nondegenerate }\right\}
$$

is an open dense subset of $\mathcal{M}^{k}$.

We could apply Theorem 1.1 to the study of the following problem:

$$
\left\{\begin{array}{l}
-\varepsilon^{2} \Delta_{g} u+u=u^{p} \quad \text { in } M, \\
u>0 \text { in } M, \\
u \in \mathrm{H}_{g}^{1}(M),
\end{array}\right.
$$

where $p>1$ if $N=2,1<p<\frac{N+2}{N-2}$ if $N \geq 3$ and $\varepsilon$ is a positive parameter. Here $\mathrm{H}_{g}^{1}(M)$ is the completion of $C^{\infty}(M)$ with respect to

$$
\|u\|_{g}^{2}:=\int_{M}\left|\nabla_{g} u\right|^{2} d \mu_{g}+\int_{M} u^{2} d \mu_{g} .
$$

It is well known that problem (1.1) has a mountain pass solution $u_{\varepsilon}$. In [2], the authors proved that $u_{\varepsilon}$ exhibits a spike layer by showing that its peak point converges to a maximum point of the scalar curvature $S_{g}$ as $\varepsilon$ goes to zero.

Received by the editors April 13, 2009.

2010 Mathematics Subject Classification. Primary 53A99, 53C21.

Key words and phrases. Scalar curvature, nondegenerate critical points.

The authors were supported by Mi.U.R. project "Metodi variazionali e topologici nello studio di fenomeni non lineari". 
Recently there have been some results on the effect of the topology and the geometry of the manifold $M$ on the number of solutions of problem (1.1). In [1] the authors showed that multiplicity of solutions to (1.1) depends on the topological properties of the manifold $M$. More precisely, they show that problem (1.1) has at least $\operatorname{cat}(M)+1$ nontrivial solutions provided $\varepsilon$ is small enough. Here cat $(M)$ denotes the Lusternik-Schnirelmann category of $M$. In [11] the author obtains the same result for a more general nonlinearity. In [5] it is shown that the number of solutions is influenced by the topology of a suitable subset of $M$ depending on the geometry of $M$.

Successively, in [6] the authors pointed out the role of the geometry in finding solutions to problem (1.1). In fact, they showed that for any $C^{1}$-stable critical point of the scalar curvature it is possible to construct a single peak solution whose peak approaches such a point as $\varepsilon$ goes to zero. Moreover, in [7] the authors constructed $K$-peaks positive or nodal solutions, whose positive and negative peaks approach $K$ different $C^{1}$-stable critical points of the scalar curvature as $\varepsilon$ goes to zero. As far as it concerns the existence of nodal solutions, we also quote [4], where the authors prove the existence of antisymmetric sign changing solutions to (1.1).

We say that $\xi_{0}$ is a $C^{1}$-stable critical point of a function $f \in \mathrm{C}^{1}(M, \mathbb{R})$ if for any $\rho>0$ there exists $\delta>0$ such that if $h \in \mathrm{C}^{1}(M, \mathbb{R})$ with

$$
\max _{\xi \in B_{g}\left(\xi_{0}, \rho\right)}\left[|f(\xi)-h(\xi)|+\left|\nabla_{g} f(\xi)-\nabla_{g} h(\xi)\right|\right] \leq \delta,
$$

then $h$ has at least one critical point $\xi \in B_{g}\left(\xi_{0}, \rho\right)$.

In particular, if $\xi_{0}$ is a strict local minimum point or a strict local maximum point of $f$ or it is a nondegenerate critical point of $f$, then $\xi_{0}$ is a $C^{1}$-stable critical point of $f$.

Recently, in [3] the authors proved that if the scalar curvature has an isolated local minimum point $\xi_{0}$, for any integer $K$ there exists a solution to (1.1) whose $K$ peaks collapse to $\xi_{0}$ as $\varepsilon$ goes to zero.

All the previous results require a sort of nondegeneracy of critical points of the scalar curvature of the manifold $(M, g)$. Theorem 1.1 allows us to claim that for a generic metric $g$, all the critical points of the scalar curvature are nondegenerate. If $\nu$ is the number of the critical points of the scalar curvature, by [6, 7] we deduce that problem (1.1) has, as $\varepsilon$ goes to zero, $\nu$ solutions with one peak, $\nu(\nu-1) / 2$ solutions with two peaks, ..., one solution with $\nu$ peaks.

Finally, since the global minimum point of the scalar curvature is nondegenerate and in particular is isolated, by [3] we deduce that the number of solutions to problem (1.1) goes to $\infty$ as $\varepsilon$ goes to zero.

The paper is organized as follows. In Section 2 we formulate the problem and prove the main result, using some technical results proved in Section 3 ,

\section{Formulation of the problem and proof of the main Result}

We denote by $\mathcal{S}^{k}$ the space of all $C^{k}$ symmetric covariant 2 -tensors on $M$. $\mathcal{S}^{k}$ is a Banach space equipped with the norm $\|\cdot\|_{k}$ defined in the following way. We fix a finite covering $\left\{V_{\alpha}\right\}_{\alpha \in L}$ of $M$ such that the closure of $V_{\alpha}$ is contained $U_{\alpha}$, where $\left\{U_{\alpha}, \psi_{\alpha}\right\}$ is the open coordinate neighbourhood. If $h \in \mathcal{S}^{k}$ we denote by $h_{i j}$ the 
components of $h$ with respect to the coordinates $\left(x_{1}, \ldots, x_{N}\right)$ on $V_{\alpha}$. We define

$$
\|h\|_{k}:=\sum_{\alpha \in L} \sum_{|\beta| \leq k} \sum_{i, j=1}^{N} \sup _{\psi_{\alpha}\left(V_{\alpha}\right)} \frac{\partial^{\beta} h_{i j}}{\partial x_{1}^{\beta_{1}} \ldots \partial x_{N}^{\beta_{N}}} .
$$

The set $\mathcal{M}^{k}$ of all $C^{k}$ Riemannian metrics on $M$ is an open set of $\mathcal{S}^{k}$.

In the following we will assume $k \geq 4$.

Given $\hat{g} \in \mathcal{M}^{k}$, it is possible to define an atlas on $M$ whose charts are $\left(B_{\hat{g}}(\xi, R)\right.$, $\left.\varphi^{-1}\right)$, where $\varphi: B(0, R) \rightarrow B_{\hat{g}}(\xi, R)$. Here $B_{\hat{g}}(\xi, R) \subset M$ is the ball centered at $\xi$ with radius $R$ given by the metric $\hat{g}$ and $B(0, R) \subset \mathbb{R}^{N}$ is the ball centered at 0 with radius $R$ in the euclidean space $\mathbb{R}^{N}$. Let $\mathcal{B}_{\rho}:=\left\{h \in \mathcal{S}^{k}:\|h\|_{k}<\rho\right\}$ be the ball centered at 0 with radius $\rho$ in $\mathcal{S}^{k}$.

For any $\xi \in M$ and $h \in \mathcal{B}_{\rho}$, with $\rho$ small enough so that $\hat{g}+h \in \mathcal{M}^{k}$, we consider the scalar curvature $S_{\hat{g}+h}(\xi)$ of $(M, \hat{g}+h)$ at the point $\xi \in M$. Given $\xi_{0} \in M$ and the chart $\left(B_{\hat{g}}\left(\xi_{0}, R\right), \varphi^{-1}\right)$ we set

$$
\widetilde{S}_{\hat{g}+h}(x):=S_{\hat{g}+h}(\varphi(x)), \quad x \in B(0, R), \quad h \in \mathcal{B}_{\rho} .
$$

Now, we introduce the $C^{1}$-map $F: \mathcal{B}_{\rho} \times B(0, R) \subset \mathcal{S}^{k} \times \mathbb{R}^{N} \rightarrow \mathbb{R}^{N}$ defined by

$$
F(h, x):=\nabla \widetilde{S}_{\hat{g}+h}(x)
$$

We shall apply to the map $F$ an abstract transversality theorem (see [8, 9, 10]). We recall it (see Theorem 1.1 of 9]) in the following.

Theorem 2.1. Let $X, Y, Z$ be three real Banach spaces and let $U \subset X, V \subset Y$ be open subsets. Let $F: V \times U \rightarrow Z$ be a $C^{k}$-map with $k \geq 1$ such that

(i) for any $y \in V, F(y, \cdot): x \rightarrow F(y, x)$ is a Fredholm map of index $l$ with $l \leq k$

(ii) $z_{0}$ is a regular value of $F$; that is, the operator $F^{\prime}\left(y_{0}, x_{0}\right): Y \times X \rightarrow Z$ is onto at any point $\left(y_{0}, x_{0}\right)$ such that $F\left(y_{0}, x_{0}\right)=z_{0}$;

(iii) the set of $x \in U$ such that $F(y, x)=z_{0}$ with $y$ in a compact set of $X$ is relatively compact in $U$.

Then the set $\left\{y \in V: z_{0}\right.$ is a regular value of $\left.F(y, \cdot)\right\}$ is a dense open subset of $V$.

By Theorem 2.1 we obtain the following result, which is crucial to deduce Theorem 1.1 .

Theorem 2.2. For any $\hat{g} \in \mathcal{M}^{k}$ the set

$$
\left\{h \in \mathcal{S}^{k}: \text { all the critical points of } S_{\hat{g}+h} \text { are nondegenerate }\right\}
$$

is an open dense subset of a suitable ball centered at 0 with radius $\rho$ in $\mathcal{S}^{k}$.

Proof. We are going to apply Theorem 2.1 to the map $F$ defined in (2.2). In this case we have $X=Z=\mathbb{R}^{N}$ and $Y=\mathcal{S}^{k}$. We choose $z_{0}=0$. Since $X$ is a finite dimensional space, it is easy to check that for any $h \in \mathcal{B}_{\rho}$ the map $x \rightarrow$ $F(h, x)$ is Fredholm of index 0, and so assumption (i) holds. Moreover, assumption (iii) immediately follows again by the fact that $X$ is a finite dimensional space. Assumption (ii) is verified in Lemma 3.4. 
Finally, we are in a position to apply Theorem 2.1. and we get that the set $\Theta\left(\xi_{0}, \rho\right):=\left\{h \in \mathcal{B}_{\rho}: F_{x}^{\prime}(h, x): \mathbb{R}^{N} \rightarrow \mathbb{R}^{N}\right.$ is invertible at any point $(h, x)$ such that $F(h, x)=0\}$

$$
=\left\{h \in \mathcal{B}_{\rho}: \text { the critical points of } S_{\hat{g}+h} \text { in } B_{\hat{g}}\left(\xi_{0}, R\right) \text { are nondegenerate }\right\}
$$

is an open dense subset of $\mathcal{B}_{\rho}$.

Now, since $M$ is compact, there exists a finite covering $\left\{B_{\hat{g}}\left(\xi_{i}, R\right)\right\}_{i=1, \ldots, \nu}$ of $M$, where $\xi_{1}, \ldots, \xi_{\nu} \in M$. For any index $i$ there exists an open dense subset $\Theta\left(\xi_{i}, \rho\right)$ (see (2.3) ) of $\mathcal{B}_{\rho}$ such that the critical points of $S_{\hat{g}+h}$ in $B_{\hat{g}}\left(\xi_{i}, R\right)$ are nondegenerate for any $h \in \Theta\left(\xi_{i}, \rho\right)$. Let $\Theta(\rho):=\bigcap_{i=1, \ldots, \nu} \Theta\left(\xi_{i}, \rho\right)$. It is immediate that $\Theta(\rho)$ is an open dense subset of $\mathcal{B}_{\rho}$ such that the critical points of $S_{\hat{g}+h}$ in $M$ are nondegenerate for any $h \in \Theta(\rho)$.

Proof of Theorem 1.1. First of all, we prove that $\mathcal{A}$ is an open set. If $\hat{g} \in \mathcal{A}$ the critical points of $S_{\hat{g}}$ are in a finite number $\xi_{1}, \ldots, \xi_{\nu}$. Let us consider the chart $\left(B_{\hat{g}}\left(\xi_{1}\right), \varphi_{1}^{-1}\right)$ and set $\widetilde{S}_{\hat{g}+h}(x):=S_{\hat{g}+h}\left(\varphi_{1}(x)\right)$. We introduce the $C^{2}$ map $F$ : $\mathcal{B}_{\rho} \times B(0, R) \rightarrow \mathbb{R}^{N}$ defined by $F(h, x):=\nabla \widetilde{S}_{\hat{g}+h}(x)$. We have $F(0,0)=0$. Moreover $F_{x}^{\prime}(0,0): \mathbb{R}^{N} \rightarrow \mathbb{R}^{N}$ is an isomorphism. Then by the implicit function theorem it is easy to deduce that locally there exists a unique $x=x(h)$ such that $F(h, x(h))=0$. Since the critical points of $S_{\hat{g}}$ are in a finite number, it is easy to check that choosing $\rho$ small enough for any $h \in \mathcal{B}_{\rho}$ has only $\nu$ critical points. They are nondegenerate because $F$ is a $C^{2}$-function.

The density of $\mathcal{A}$ follows by Theorem 2.2,

\section{TeChNicAl LEMmas}

Let us recall the definition of the scalar curvature $S_{g}(\xi)$ of the metric $g$ at the point $\xi$. Let $\xi_{0} \in M$ be fixed. Given a coordinate system, the scalar curvature in a point $\xi(x)$ belonging to $B_{g}\left(\xi_{0}, R\right)$ can be expressed as follows:

$$
\widetilde{S}_{g}(x)=S_{g}(\xi(x))=g^{a b}\left(\partial_{c} \Gamma_{a b}^{c}-\partial_{b} \Gamma_{a c}^{c}+\Gamma_{a b}^{c} \Gamma_{c d}^{d}-\Gamma_{a c}^{d} \Gamma_{b d}^{c}\right) .
$$

Here $\Gamma_{i j}^{l}$ denotes the Christoffel symbol with respect to $g$, namely

$$
\Gamma_{i j}^{l}=\Gamma_{i j}^{l}(g, x)=\frac{1}{2} g^{l m}\left(\partial_{j} g_{m i}+\partial_{i} g_{m j}-\partial_{m} g_{i j}\right) .
$$

We are using the Einstein summation convention; i.e. when an index variable appears twice in a single term, once in an upper (superscript) and once in a lower (subscript) position, it implies that we are summing over all of its possible values.

We are going to compute the first and second derivatives of $\Gamma_{i j}^{l}(g, x)$ with respect to $x$.

It is useful to introduce the following notation. Let

$$
G_{i j k}=G_{i j k}(g, x)=\partial_{i} g_{k j}+\partial_{j} g_{k i}-\partial_{k} g_{i j} .
$$

By (3.2) and (3.3) we deduce

$$
\Gamma_{i j}^{l}(g, x)=\frac{1}{2} g^{l k} G_{i j k}(g, x) .
$$


Lemma 3.1. It holds that for any $x \in B(0, R)$ and for any $h \in \mathcal{B}_{\rho}$

$$
\begin{aligned}
& \partial_{\alpha} \Gamma_{i j}^{l}(g, x)=\frac{1}{2} \partial_{\alpha} g^{l k} G_{i j k}(g, x)+\frac{1}{2} g^{l k} \partial_{\alpha} G_{i j k}(g, x), \\
& \partial_{\alpha \beta}^{2} \Gamma_{i j}^{l}(g, x)=\frac{1}{2} \partial_{\alpha \beta}^{2} g^{l k} G_{i j k}(g, x)+\frac{1}{2} g^{l k} \partial_{\alpha \beta}^{2} G_{i j k}(g, x) \\
& +\partial_{\alpha} g^{l k} \partial_{\beta} G_{i j k}(g, x)+\partial_{\beta} g^{l k} \partial_{\alpha} G_{i j k}(g, x), \\
& D_{g} \Gamma_{i j}^{l}(g, x)[h]=\frac{1}{2} g^{l k} G_{i j k}(h, x)-\frac{1}{2} g^{l s} h_{s t} g^{t k} G_{i j k}(g, x), \\
& \partial_{\alpha} D_{g} \Gamma_{i j}^{l}(g, x)[h]=\frac{1}{2} \partial_{\alpha} g^{l k} G_{i j k}(h, x)+\frac{1}{2} g^{l k} \partial_{\alpha} G_{i j k}(h, x) \\
& -\frac{1}{2} \partial_{\alpha}\left(g^{l s} h_{s t} g^{t k}\right) G_{i j k}(g, x)-\frac{1}{2} g^{l s} h_{s t} g^{t k} \partial_{\alpha} G_{i j k}(g, x) \\
& =D_{g} \partial_{\alpha} \Gamma_{i j}^{l}(g, x)[h], \\
& \partial_{\alpha \beta}^{2} D_{g} \Gamma_{i j}^{l}(g, x)[h]=\frac{1}{2} \partial_{\alpha \beta}^{2} g^{l k} G_{i j k}(h, x)+\frac{1}{2} \partial_{\alpha} g^{l k} \partial_{\beta} G_{i j k}(h, x) \\
& +\frac{1}{2} \partial_{\beta} g^{l k} \partial_{\alpha} G_{i j k}(h, x)+\frac{1}{2} g^{l k} \partial_{\alpha \beta}^{2} G_{i j k}(h, x) \\
& -\frac{1}{2} \partial_{\alpha \beta}^{2}\left(g^{l s} h_{s t} g^{t k}\right) G_{i j k}(g, x)-\frac{1}{2} \partial_{\alpha}\left(g^{l s} h_{s t} g^{t k}\right) \partial_{\beta} G_{i j k}(g, x) \\
& -\frac{1}{2} g^{l s} h_{s t} g^{t k} \partial_{\alpha \beta}^{2} G_{i j k}(g, x)-\frac{1}{2} \partial_{\beta}\left(g^{l s} h_{s t} g^{t k}\right) \partial_{\alpha} G_{i j k}(g, x) \\
& =D_{g} \partial_{\alpha \beta}^{2} \Gamma_{i j}^{l}(g, x)[h] .
\end{aligned}
$$

Now, we can evaluate the derivative of the scalar curvature.

Lemma 3.2. It holds that for any $x \in B(0, R)$ and for any $h \in \mathcal{B}_{\rho}$

$$
\begin{aligned}
& \partial_{\alpha} \widetilde{S}_{g}(x)=\partial_{\alpha} g^{a b}\left(\partial_{c} \Gamma_{a b}^{c}-\partial_{b} \Gamma_{a c}^{c}+\Gamma_{a b}^{c} \Gamma_{c d}^{d}-\Gamma_{a c}^{d} \Gamma_{b d}^{c}\right) \\
& +g^{a b}\left(\partial_{\alpha c}^{2} \Gamma_{a b}^{c}-\partial_{\alpha b}^{2} \Gamma_{a c}^{c}+\partial_{\alpha} \Gamma_{a b}^{c} \Gamma_{c d}^{d}+\Gamma_{a b}^{c} \partial_{\alpha} \Gamma_{c d}^{d}-\partial_{\alpha} \Gamma_{a c}^{d} \Gamma_{b d}^{c}-\Gamma_{a c}^{d} \partial_{\alpha} \Gamma_{b d}^{c}\right)
\end{aligned}
$$

and

$$
\begin{aligned}
& \partial_{\alpha} D_{g} \widetilde{S}_{g}(x)[h]=D_{g} \partial_{\alpha} \widetilde{S}_{g}(x)[h] \\
& =\partial_{\alpha}\left(g^{a t} h_{t s} g^{s b}\right)\left(\partial_{c} \Gamma_{a b}^{c}-\partial_{b} \Gamma_{a c}^{c}+\Gamma_{a b}^{c} \Gamma_{c d}^{d}-\Gamma_{a c}^{d} \Gamma_{b d}^{c}\right) \\
& +g^{a b}\left(\partial_{\alpha c}^{2} D_{g} \Gamma_{a b}^{c}[h]-\partial_{\alpha b}^{2} D_{g} \Gamma_{a c}^{c}[h]+\partial_{\alpha} D_{g} \Gamma_{a b}^{c}[h] \Gamma_{c d}^{d}+\partial_{\alpha} \Gamma_{a b}^{c} D_{g} \Gamma_{c d}^{d}[h]\right. \\
& +D_{g} \Gamma_{a b}^{c}[h] \partial_{\alpha} \Gamma_{c d}^{d}+\Gamma_{a b}^{c} \partial_{\alpha} D_{g} \Gamma_{c d}^{d}[h]-\partial_{\alpha} D_{g} \Gamma_{a c}^{d}[h] \Gamma_{b d}^{c}-\partial_{\alpha} \Gamma_{a c}^{d} D_{g} \Gamma_{b d}^{c}[h] \\
& \left.-D_{g} \Gamma_{a c}^{d}[h] \partial_{\alpha} \Gamma_{b d}^{c}-\Gamma_{a c}^{d} \partial_{\alpha} D_{g} \Gamma_{b d}^{c}[h]\right) .
\end{aligned}
$$

Given the metric $g=\hat{g}+h$ with $h \in \mathcal{B}_{\rho}$ and a point $\xi \in B_{g}\left(\xi_{0}, R\right)$, let us consider the local normal coordinates on the Riemannian manifold $(M, g)$ given by the exponential map $\exp _{\xi}(z)$. Let $g_{\xi}$ be the metric $g$ read in $B(0, R)$ through the 
normal coordinates. In particular

$$
g_{\xi}^{i j}(0)=g_{\xi_{i j}}(0)=\delta_{i j} \quad \text { and } \quad \frac{\partial g_{\xi}^{i j}}{z_{k}}(0)=\frac{\partial g_{\xi_{i j}}}{z_{k}}(0)=0,
$$

which implies $\Gamma_{i j}^{k}\left(g_{\xi}, 0\right)=0$ for any indexes $i, j$ and $k$. Here $g_{\xi}^{i j}(z)$ is the inverse matrix of $g_{\xi_{i j}}(z)$ and $\delta_{i j}$ is the Kronecker symbol.

Let us evaluate the derivatives of Lemma 3.1 at the point $\xi$ using the normal coordinates.

Lemma 3.3. It holds that

$$
\partial_{\alpha} \Gamma_{i j}^{l}\left(g_{\xi}, 0\right)=\frac{1}{2} \partial_{\alpha} G_{i j l}\left(g_{\xi}, 0\right)
$$

and

$$
\partial_{\alpha \beta}^{2} \Gamma_{i j}^{l}\left(g_{\xi}, 0\right)=\frac{1}{2} \partial_{\alpha \beta}^{2} G_{i j l}\left(g_{\xi}, 0\right) .
$$

Moreover

$$
\begin{gathered}
D_{g} \Gamma_{i j}^{l}\left(g_{\xi}, 0\right)[h]=\frac{1}{2} G_{i j l}(h, 0), \\
\partial_{\alpha} D_{g} \Gamma_{i j}^{l}\left(g_{\xi}, 0\right)[h]=\frac{1}{2} \partial_{\alpha} G_{i j l}(h, 0)-\frac{1}{2} h_{l k}(0) \partial_{\alpha} G_{i j k}\left(g_{\xi}, 0\right),
\end{gathered}
$$

and

$$
\begin{aligned}
\partial_{\alpha \beta}^{2} D_{g} \Gamma_{i j}^{l}(g)[h] & =\frac{1}{2} \partial_{\alpha \beta}^{2} g^{l k}(0) G_{i j k}(h, 0)+\frac{1}{2} \partial_{\alpha \beta}^{2} G_{i j l}(h, 0) \\
& -\frac{1}{2} \sum_{k} \partial_{\alpha} h_{l k}(0) \partial_{\beta} G_{i j k}\left(g_{\xi}, 0\right)-\frac{1}{2} \sum_{k} \partial_{\beta} h_{l k}(0) \partial_{\alpha} G_{i j k}\left(g_{\xi}, 0\right) \\
& -\frac{1}{2} \sum_{k} h_{l k}(0) \partial_{\alpha \beta}^{2} G_{i j k}\left(g_{\xi}, 0\right) .
\end{aligned}
$$

Lemma 3.4. The map $(h, x) \rightarrow F_{h}^{\prime}(\tilde{h}, \tilde{x})[h]+F_{x}^{\prime}(\tilde{h}, \tilde{x}) x$ is onto on $\mathbb{R}^{N}$ for any $(\tilde{h}, \tilde{x})$ such that $F(\tilde{h}, \tilde{x})=0$.

Proof. Let $\hat{g}+h$ with $h \in \mathcal{B}_{\rho} \subset \mathcal{S}^{k}$ with $k \geq 4$. By (3.5) of Lemma 3.2 we have that the function $F(h, x)=\nabla_{x} \widetilde{S}_{\hat{g}+h}(x)$ defined in (2.2) is of class $C^{2}$. Let $(\tilde{h}, \tilde{x})$ be such that $F(\tilde{h}, \tilde{x})=0$. By Lemma 3.2 we get

$$
F_{h}^{\prime}(\tilde{h}, \tilde{x})[h]=\left(D_{h} \partial_{1} \widetilde{S}_{\hat{g}+\tilde{h}}(\tilde{x})[h], \ldots, D_{h} \partial_{N} \widetilde{S}_{\hat{g}+\tilde{h}}(\tilde{x})[h]\right) .
$$

We will prove that the map $F_{h}^{\prime}(\tilde{h}, \tilde{x}): \mathcal{S}^{k} \rightarrow \mathbb{R}^{N}$ is onto. More precisely, we are going to show that given $e_{1}, \ldots, e_{N}$ the canonical base in $\mathbb{R}^{N}$, for any $i=1, \ldots, N$

$$
\text { there exists } h \in \mathcal{S}^{k} \text { such that } F_{h}^{\prime}(\tilde{h}, \tilde{x})[h]=e_{i} \text {. }
$$

The claim will follow immediately.

We point out that the ontoness of the map $h \rightarrow F_{h}^{\prime}(\tilde{h}, \tilde{x})[h]$ is invariant with respect to a change of variable $x=\psi(z)$, where $\psi$ is a diffeomorphism. Therefore, to show (3.13), we calculate $D_{h} \partial_{\alpha} \widetilde{S}_{\hat{g}+\tilde{h}}(\tilde{x})[h]$ by choosing the normal coordinates on the Riemannian manifold $(M, \hat{g}+\tilde{h})$ given by the exponential map $\exp _{\tilde{\xi}}(z)$, where $\tilde{\xi}$ corresponds to $\tilde{x}$. 
We choose $h \in \mathcal{S}^{k}$ such that the map $z \rightarrow h_{i j}\left(\exp _{\tilde{\xi}}(z)\right)$, with its first and second derivatives, is vanishing at the point 0 , for any indexes $i$ and $j$. By Lemma 3.3 . taking into account the definition of $G_{i j k}$ given in (3.3), we deduce that

$$
\partial_{\alpha} \Gamma_{i j}^{l}\left(g_{\xi}, 0\right)=D_{g} \Gamma_{i j}^{l}\left(g_{\xi}, 0\right)[h]=\partial_{\alpha} D_{g} \Gamma_{i j}^{l}\left(g_{\xi}, 0\right)[h]=0
$$

and

$$
\partial_{\alpha \beta}^{2} \Gamma_{i j}^{l}\left(g_{\xi}, 0\right)[h]=\frac{1}{2} \partial_{\alpha \beta}^{2} G_{i j l}\left(g_{\xi}, 0\right), \quad \partial_{\alpha \beta}^{2} D_{g} \Gamma_{i j}^{l}\left(g_{\xi}, 0\right)[h]=\frac{1}{2} \partial_{\alpha \beta}^{2} G_{i j l}(h, 0) .
$$

Then by (3.6), (3.14) and (3.15) we deduce

$$
\begin{aligned}
& \partial_{\alpha} D_{g} \widetilde{S}_{g}(x)[h]=\partial_{\alpha c}^{2} D_{g} \Gamma_{a a}^{c}[h]-\partial_{\alpha a}^{2} D_{g} \Gamma_{a c}^{c}[h] \\
& =\frac{1}{2} \sum_{a, c}\left\{\partial_{\alpha c}^{2} G_{a a c}\left(g_{\xi}, 0\right)[h]-\partial_{\alpha a}^{2} G_{a c c}\left(g_{\xi}, 0\right)[h]\right\} \\
& =\sum_{a, c}\left\{\partial_{\alpha a c}^{3} h_{a c}(0)-\partial_{\alpha a a}^{3} h_{c c}(0)\right\} .
\end{aligned}
$$

Let us prove (3.13) when $i=1$. Let us choose $h \in \mathcal{S}^{k}$ such that

$$
h_{11}(z)=-\frac{1}{2} z_{1} z_{2}^{2} \quad \text { and } \quad h_{i j}(z)=0 \quad \text { for all the other indexes. }
$$

An easy computation shows that

$$
\sum_{a, c}\left\{\partial_{1 a c}^{3} h_{a c}(0)-\partial_{1 a a}^{3} h_{c c}(0)\right\}=-\partial_{122}^{3} h_{11}(0)=1
$$

and

$$
\sum_{a, c}\left\{\partial_{\alpha a c}^{3} h_{a c}(0)-\partial_{\alpha a a}^{3} h_{c c}(0)\right\}=0 \quad \text { if } \quad \alpha \neq 1 .
$$

Finally, by (3.12) and (3.16) we deduce that $F_{h}^{\prime}(\tilde{h}, \tilde{x})[h]=e_{1}$. We argue in a similar way to prove (3.13) when $i=2, \ldots, N$. This concludes the proof.

\section{REFERENCES}

[1] Benci, V.; Bonanno, C.; Micheletti, A.M., On the multiplicity of solutions of a nonlinear elliptic problem on Riemannian manifolds. J. Funct. Anal. 252 (2007), no. 2, 464-489. MR2360924 (2008m:35091)

[2] Byeon, J.; PARK, J. Singularly perturbed nonlinear elliptic problems on manifolds. Calc. Var. Partial Differential Equations 24 (2005), no. 4, 459-477. MR2180862 (2007i:58007)

[3] Dancer, E.N.; Micheletti A.M.; Pistoia, A., Multipeak solutions for some singularly perturbed nonlinear elliptic problems on Riemannian manifolds. Manuscripta Math. 128 (2009), no. 2, 163-193. MR2471314

[4] Ghimenti, M.; Micheletti A.M., On the number of nodal solutions for a nonlinear elliptic problem on symmetric Riemannian manifolds. Electronic Journal of Differential Equations (to appear).

[5] Hirano, N., Multiple existence of solutions for a nonlinear elliptic problem on a Riemannian manifold. Nonlinear Anal. 70 (2009), no. 2, 671-692. MR2468411

[6] Micheletti A.M.; Pistoia, A., The role of the scalar curvature in a nonlinear elliptic problem on Riemannian manifolds. Calculus of Variations PDE 34 (2009), no. 2, 233-265. MR2448651 (2009k:53084)

[7] Micheletti A.M.; Pistoia, A., Nodal solutions for a singularly perturbed nonlinear elliptic problem on Riemannian manifolds. Advanced Nonlinear Studies 9 (2009), 565-577. MR 2536955 
[8] Quinn, F., Transversal approximation on Banach manifolds. 1970 Global Analysis (Proc. Sympos. Pure Math., Vol. XV, Berkeley, Calif., 1968), pp. 213-222, Amer. Math. Soc., Providence, R.I. MR0264713 (41:9304)

[9] Saut, J.-C.; Temam, R., Generic properties of nonlinear boundary value problems. Comm. Partial Differential Equations 4 (1979), no. 3, 293-319. MR/522714(80i:35080)

[10] Uhlenbeck, K., Generic properties of eigenfunctions. Amer. J. Math. 98 (1976), no. 4, 1059-1078. MR0464332 (57:4264)

[11] Visetti, D., Multiplicity of solutions of a zero-mass nonlinear equation on a Riemannian manifold. J. Differential Equations 245 (2008), no. 9, 2397-2439. MR2455770(2009m:35169)

Dipartimento di Matematica Applicata "U. Dini", Università di Pisa, via F. BuonarRoti 1/C, 56100 Pisa, ItAly

E-mail address: a.micheletti@dma.unipi.it

Dipartimento di Metodi e Modelli Matematici, Università di Roma "La Sapienza", via Antonio Scarpa 16, 00161 Roma, Italy

E-mail address: pistoia@dmmm.uniroma1.it 